\title{
Roles of the Nucleus Accumbens and Amygdala in the Acquisition and Expression of Ethanol-Conditioned Behavior in Mice
}

\author{
Christina M. Gremel and Christopher L. Cunningham \\ Department of Behavioral Neuroscience and Portland Alcohol Research Center, Oregon Health \& Science University, Portland, Oregon 97239-3098
}

\begin{abstract}
Although progress has been made identifying the neural areas underlying the primary reinforcing effects of ethanol, few studies have examined the neural areas mediating ethanol-induced conditioned effects. Previous work using the conditioned place preference (CPP) procedure implicates the ventral tegmental area (VTA) (Bechtholt and Cunningham, 2005), but the downstream neural areas modulating the conditioned rewarding effects of ethanol have not been identified. Although the nucleus accumbens (Acb) and the amygdala (Amy), which both receive dopamine innervation from the VTA, have been implicated in the primary reinforcing effects of ethanol, the roles these areas play in ethanol-conditioned behaviors are unknown. In the present set of experiments, we use the CPP procedure along with selective electrolytic lesions to examine the neural areas underlying the acquisition and expression of ethanol conditioned behavior. In the acquisition experiment, male DBA/2J mice received bilateral lesions of the Acb or Amy before CPP training. In the expression experiments, mice received bilateral lesions of the Acb, Acb shell, Acb core, and Amy, or unilateral lesions of the Amy after training but before testing. Lesions of the Acb and Amy before training disrupted acquisition and expression of ethanol CPP. However, when given after training, only lesions of the Amy disrupted expression, whereas lesions of the Acb core facilitated loss of responding, of ethanol CPP. For the first time, these results demonstrate the role of the Acb and Amy in the acquisition and expression of ethanol-induced conditioned reward.
\end{abstract}

Key words: conditioned place preference; conditioned reinforcement; locomotor activity; lesion; inbred mice (DBA/2); accumbens

\section{Introduction}

Ethanol-seeking behavior is strongly influenced by learning about the pavlovian relationship between environmental cues and the rewarding or aversive effects of ethanol. Such learning is important because it can alter physiological/behavioral responses, motivational states (e.g., craving), or expectancies that affect the probability, vigor, or cue-directed nature of ethanolseeking behavior (Krank, 1989, 2003; Cunningham, 1994, 1998; Corbit and Janak, 2007). Pavlovian learning may be especially involved in triggering relapse to ethanol seeking after periods of abstinence or extinction (Krank and Wall, 1990; Katner and Weiss, 1999; Ciccocioppo et al., 2001, 2002, 2003; Nie and Janak, 2003; Zironi et al., 2006; Dayas et al., 2007). Despite the prevalence of alcohol abuse and dependence, surprisingly little is known about the neural areas that mediate the acquisition and expression of ethanol-seeking behaviors.

Substantial work has been done to identify and characterize

Received June 13, 2007; revised Dec. 4, 2007; accepted Dec. 5, 2007

This work was supported by National Institutes of Health/National Institute on Alcohol Abuse and Alcoholism Grants AA016041, AA007468, and AA007702. We thank Peter Groblewski and Charlene Voorhees for comments on this paper. Experiments within this manuscript comply with the current laws of the United States of America.

Correspondence should be addressed to Christina M. Gremel, Department of Behavioral Neuroscience, L470, Oregon Health \& Science University, 3181 SW Sam Jackson Park Road, Portland, OR 97239-3098. E-mail: gremel@@ohsu.edu.

DOI:10.1523/JNEUROSCI.4520-07.2008

Copyright $\odot 2008$ Society for Neuroscience $\quad$ 0270-6474/08/281076-09\$15.00/0 brain regions involved in the primary rewarding effects of ethanol and other abused drugs (Koob and Le Moal, 2006). Moreover, significant effort has been devoted to identifying brain areas that regulate conditioned effects of cues associated with cocaine (Everitt and Robbins, 2005; Di Ciano and Everitt, 2005; Weiss, 2005). In contrast, very few studies have addressed the neurocircuitry underlying conditioned effects of cues paired with ethanol. Two recent studies reported increased Fos expression in brain areas activated by exposure to cues previously associated with ethanol either as a discriminative stimulus in an operant selfadministration task (Dayas et al., 2007) or as a conditioned stimulus (CS+) in a pavlovian procedure (Hill et al., 2007). Only one previous study, however, has addressed the functional role of any brain area in the expression of behavior elicited by an ethanol-paired stimulus. That study, which used a conditioned place preference (CPP) procedure, showed that microinfusion of a nonselective opioid antagonist or $\mathrm{GABA}_{\mathrm{B}}$ agonist into the ventral tegmental area (VTA) reduced CPP expression (Bechtholt and Cunningham, 2005), suggesting that VTA dopamine (DA) neurons might play as important a role in the expression of ethanol-induced conditioned effects as they have been shown previously to play in the direct effects of ethanol (Gessa et al., 1985; Brodie et al., 1990, 1999; Samson and Hodge, 1996; Gatto et al., 1994; Rodd et al., 2004).

The downstream targets of ethanol-induced conditioned changes in VTA DA neurons are unknown, although previous studies strongly suggest that both the nucleus accumbens (Acb) 
and central nucleus of the amygdala (CE) might be involved. For example, microdialysis studies have shown that ethanol increases DA release in Acb (Di Chiara and Imperato, 1988) and in CE (Yoshimoto et al., 2000). Moreover, microinfusion of opioid or GABA receptor antagonists into Acb and CE reduces ethanol self-administration (Hyytiä and Koob, 1995). Although both areas have been implicated in the primary reinforcing effects of ethanol, their roles in the conditioned rewarding effects of ethanol remain unknown. In the present studies, we use selective electrolytic lesions in a well established model of ethanolconditioned behavior in mice, CPP (Cunningham et al., 2006a,b), to examine whether the Acb and amygdala (Amy) influence the acquisition and expression of this behavior.

\section{Materials and Methods}

Subjects

Male DBA/2J $(n=289)$ mice were obtained from The Jackson Laboratory (Bar Harbor, ME) at 6-7 weeks of age. Mice were selected for these studies instead of rats because of the substantial difficulty in establishing a reliable ethanol CPP in rats (Fidler et al., 2004; Tzschentke, 2007), which may reflect a reduced sensitivity to the rewarding effect of ethanol as indexed by this procedure (Cunningham et al., 1993). In contrast, many previous studies have shown that DBA/2J mice develop a strong preference for ethanol-paired cues at a dose of $2 \mathrm{~g} / \mathrm{kg}$ (Cunningham et al., 2003, 2006a). Animals were housed in groups of four on a Thoren rack (Thoren Caging Systems, Hazleton, PA) in polycarbonate cages. Animals were kept at an ambient temperature of $21 \pm 1^{\circ} \mathrm{C}$ on a $12 \mathrm{~h}$ light/dark cycle (lights on at 7:00 A.M.). Experiments were performed during the light portion of the cycle beginning at 1:00 P.M. LabDiet (Richmond, IN) rodent chow and bottled water were continuously available in the home cage. The National Institutes of Health Principles of Laboratory Animal Care were followed in conducting these studies, and the protocol was approved by the Oregon Health and Science University Institutional Animal Care and Use Committee.

\section{Surgery}

Electrolytic lesions were administered to examine the role of the Amy and $\mathrm{Acb}$ in the acquisition and expression of ethanol CPP. We chose electrolytic over excitotoxic lesions in light of previous studies showing differences across mouse genotypes in the induction of lesions with excitotoxic agents in the striatum and hippocampus (Schauwecker and Steward, 1997; Schauwecker, 2005) and the paucity of excitotoxic lesion studies in DBA/2J mice. Moreover, we chose not to use temporary chemical inactivation of these nuclei to avoid potential problems associated with multiple injections into mouse brain and to control for the number and type of intracranial manipulations between acquisition and expression experiments. Mice were fully anesthetized with a mixture $(0.1 \mathrm{ml} / 25 \mathrm{~g})$ containing ketamine $(30.0 \mathrm{mg} / \mathrm{ml})$ and xylazine $(3.0 \mathrm{mg} / \mathrm{ml})$. Electrodes (Rhodes Medical Instruments, Woodland Hills, CA) were lowered through small burr holes in the skull to a specified depth under stereotaxic guidance (model 1900; David Kopf Instruments, Tujunga, CA). For bilateral and unilateral (Uni) lesions of the Amy, electrodes were positioned according to the mouse brain atlas (Paxinos and Franklin, 2001) [from bregma in mm: anterior $(\mathrm{A})-1.46$, lateral $(\mathrm{L}) \pm 2.75$, ventral $(\mathrm{V})$ -5.0], and 0.5 mA of current (model 3500; Ugo Basile, Schwenksville, $\mathrm{PA}$ ) was passed for $10 \mathrm{~s}$, whereas for lesions of the Acb (from bregma in $\mathrm{mm}$ : A +1.2; $\mathrm{L} \pm 1.0 ; \mathrm{V}-4.5), 0.5 \mathrm{~mA}$ of current was passed for $15 \mathrm{~s}$. Specific lesions of the Acb core (AcbC) and Acb shell (AcbSh) were induced by electrodes positioned at $\mathrm{A}+1.42, \mathrm{~L} \pm 1.0, \mathrm{~V}-4.25$ and $\mathrm{A}$ $+1.54, \mathrm{~L} \pm 1.0, \mathrm{~V}-4.75$, respectively, and $0.5 \mathrm{~mA}$ of current was passed for $5 \mathrm{~s}$. For sham-lesioned mice, electrodes were lowered into the Amy, Acb, AcbC, AcbSh, or fimbria fornix ${ }^{a}$ although no current was passed.

${ }^{a}$ Initially, a group of subjects (not included in total $n$ ) received lesions of the fimbria fornix consisting of a midline lesion (from bregma: $\mathrm{A}-0.5, \mathrm{~L} 0.0, \mathrm{~V}-2.2$ ) of $1.0 \mathrm{~mA}$ of current passed for 20 or $30 \mathrm{~s}$. All subjects with fimbria fornix lesions were excluded because the lesions either extended into the surrounding cortices or verification was impossible because of the difficulty involved in maintaining the integrity of the slice. However, sham subjects with electrodes lowered into the fimbria fornix were included because analyses did not reveal any differences between sham sites.
Order of administered lesions (i.e., left hemisphere first, right hemisphere first) and side of lesion for the unilateral lesions (left vs right) were counterbalanced across subjects. Mice were allowed 4-12 d of recovery before the start of conditioning trials or testing. Additionally, to control for possible effects of recovery time, we counterbalanced the number of recovery days between lesion groups.

\section{Apparatus}

A detailed description and picture of the apparatus has been published previously (Cunningham et al., 2006a). Briefly, the apparatus consisted of 12 identical acrylic and aluminum boxes $(30 \times 15 \times 15 \mathrm{~cm})$ enclosed in individual ventilated, light- and sound-attenuating chambers (model E10-20; Coulbourn Instruments, Allentown, PA). Six sets of infrared light sources and photodetectors mounted $2.2 \mathrm{~cm}$ above the floor at $5 \mathrm{~cm}$ intervals along the long wall of the box detected general activity, location in the box, and time spent on each side of the chamber $(10 \mathrm{~ms}$ resolution).

CSs consisted of two interchangeable distinctive floor halves placed beneath each box. The hole floor was made from perforated stainless steel sheet metal (16 gauge) containing $6.4 \mathrm{~mm}$ round holes on $9.5 \mathrm{~mm}$ staggered centers. The grid floor was constructed from $2.3 \mathrm{~mm}$ stainless steel rods mounted $6.4 \mathrm{~mm}$ apart in acrylic rails. This floor texture combination was selected on the basis of many previous studies demonstrating that drug-naive control DBA/2J mice spend approximately half their time on each floor type during choice tests (Cunningham et al., 2003). The inside and floors of the box were wiped with a damp sponge, and the litter paper underneath the flooring was changed between animals.

\section{Drugs}

Ethanol $(95 \%)$ was diluted in $0.9 \%$ saline $(20 \% \mathrm{v} / \mathrm{v})$ and administered at a dose of $2 \mathrm{~g} / \mathrm{kg}(12.5 \mathrm{ml} / \mathrm{kg})$. In previous experiments, this ethanol dose has reliably induced a strong CPP in DBA/2J mice (Cunningham et al., 2003) without detrimental behavioral effects of repeated injections at this dose or concentration (Cunningham et al., 1997). Saline was administered in a volume of $12.5 \mathrm{ml} / \mathrm{kg}$.

\section{Experimental design}

In experiment $1(n=72)$, mice were allowed to acclimate to their surroundings for $2 \mathrm{~d}$ before undergoing surgery. Mice were then given bilateral lesions of the Amy or Acb or sham lesions. Surgeries were conducted across $8 \mathrm{~d}$, with an additional $4 \mathrm{~d}$ of recovery before subsequent ethanol place conditioning and testing (Table 1 ). In experiment 2 , which was performed in two replications (total $n=169$ ), mice were lesioned after ethanol place conditioning but before testing. Mice in the first replication received sham, Acb, or Amy lesions. Based on the results from the first replicate, we included a unilateral Amy lesion group along with sham and Amy groups in a second replicate to determine whether one intact Amy was sufficient for expression of a CPP. In experiment $3(n=48)$, to examine the role of Acb subregions in expression of $\mathrm{CPP}$, mice received specific AcbC, AcbSh, or sham lesions after ethanol place conditioning. For experiments 2 and 3, ethanol place conditioning began 2 weeks after arrival. Surgical procedures began $24 \mathrm{~h}$ after the last conditioning trial and spanned a course of $8 \mathrm{~d}$. An additional $4 \mathrm{~d}$ of recovery were allowed, with testing beginning a total time of $13 \mathrm{~d}$ after the last conditioning trial.

\section{Procedure}

Each experiment involved three phases: pretest or habituation (one session), conditioning (eight sessions), and one test session. Each animal was given an intraperitoneal injection immediately before being placed in the center of the apparatus for each session.

Pretest. A 30 min pretest was conducted in experiment 1 to determine whether the lesions or surgical procedure affected initial bias for the tactile cues. Subjects were weighed and given an intraperitoneal injection of saline just before placement in the apparatus on a test floor containing both floor types (grid and hole). A pretest was also conducted for the first replication of experiment 2 and experiment 3 . Subjects in the second replication of experiment 2 underwent a 5 min habituation trial in which they were given an injection of saline and exposed to the apparatus on a smooth paper floor to reduce the novelty and stress associated with handling, injection, and exposure to the apparatus. 
Conditioning. Mice were randomly assigned to groups that received a specific lesion type: Amy, Uni (unilateral Amy), Acb, AcbC, AcbSh, or sham lesions (Table 1). Within each lesion group, mice were randomly assigned to one of two conditioning subgroups (Grid+ or Grid-) using an unbiased, one-compartment procedure (Cunningham et al., 2003, 2006a). Both subgroups were exposed to a differential pavlovian conditioning procedure in which they received four $\mathrm{CS}+$ and four $\mathrm{CS}-$ trials. Mice in the Grid+ condition received ethanol paired with the grid floor $(\mathrm{CS}+)$ and saline paired with the hole floor (CS-). Mice in the Grid- condition received ethanol paired with the hole floor $(\mathrm{CS}+)$ and saline paired with the grid floor (CS-). Each animal received four 5-min conditioning trials of each type on alternating days over a period of $8 \mathrm{~d}$, with the presentation order of CS + and CS - trials counterbalanced within each group.

Place preference test. The test began $24 \mathrm{~h}$ after the last conditioning trial for experiment 1 or $13 \mathrm{~d}$ (surgery and recovery) after the last conditioning trial for experiment 2 . The test duration was 30 min for experiments 1 and 2. Test duration was lengthened to $60 \mathrm{~min}$ for experiment 3 based on unpublished pilot data suggesting that deficits in Acb modulation of ethanol CPP become apparent during a longer test session. Mice in all experiments were given a saline injection before the test session. Immediately after the injection, subjects were placed in the center of the apparatus with both test floors (half grid/half hole). Position (i.e., left vs right) of each floor type was counterbalanced within subgroups.

\section{Histology}

Immediately after testing, animals were given an overdose of sodium pentobarbital $(150 \mathrm{mg} / \mathrm{kg})$, and heads were removed and postfixed in $4 \%$ $(\mathrm{w} / \mathrm{v})$ paraformaldehyde in isotonic sodium PBS. After $24 \mathrm{~h}$, brains were dissected from the skull and placed into a solution of $2 \%$ paraformaldehyde for an additional $24 \mathrm{~h}$. After fixation, brains were cryoprotected using a sucrose saturation procedure consisting of $24 \mathrm{~h}$ incubations in $20 \%$ and then $30 \%$ sucrose in PBS and $0.1 \% \mathrm{NaN}_{3}$. Frozen $40 \mu \mathrm{m} \mathrm{sec-}$ tions were collected through, as well as caudal and rostral to, the lesion site. Slices were directly mounted onto slides and thionin stained.

Inclusion criteria for the Amy and Uni groups included lesions of at least two of the following areas in each hemisphere lesioned: CE, basolateral nucleus (BLA), basolateral amygdala ventral, basomedial nucleus, basomedial amygdala posterior, medial amygdala posterior dorsal and medial, amygdalohippocampal area, and bed nucleus stria terminalis intra-amygdaloid nucleus. In the Acb group, lesions of the AcbC and AcbSh were included. Additionally, Acb and AcbC lesions that extended into the anterior commissure (anterior) were included given that the anterior commissure is surrounded by the AcbC. Subjects in the AcbSh group were included if most ( $\sim 90 \%$, blind determination) of the lesion was located in the shell region. For the AcbC group, mice were included if $\sim 90 \%$ (blind determination) of the lesion encompassed the core. The possibility of some lesion spread extending into either the core or the shell for the AcbSh or AcbC groups, respectively, could not be avoided.

\section{Data analysis}

The primary dependent variable was the amount of time spent on the grid floor during the test session. In this unbiased design, the magnitude of the difference in time spent on the grid floor between the Grid+ and Grid- conditioning subgroups is indicative of CPP. For a more complete discussion of dependent variables used in place conditioning studies, see Cunningham et al. (2003). Data from each experiment were evaluated separately by ANOVA with the $\alpha$ level set at 0.05 . To control overall $\alpha$ level within each experiment, $p$ values were Bonferroni's corrected for the number of post hoc comparisons between group means. Paired $t$ tests were performed when appropriate. Lesion group, conditioning sub- group (Grid+ vs Grid-), hemisphere (left vs right), and sham site were treated as between-group factors, whereas trial type (CS+ vs CS-) was treated as a within-subject factor. Replication was included as an additional between-group factor in experiment 2 analyses involving only the Amy and sham groups.

\section{Results}

\section{Subject removal}

Initially, data from two subjects in experiment 2 were removed because of procedural errors during conditioning. Both subjects had received lesions of the Amy. Furthermore, an additional 18 subjects were removed from analyses because they were killed during recovery from surgery as a result of poor health. Of those removed in experiment 1, seven subjects were from the Amy group and one subject was from the Acb group, whereas of those killed in experiment 2, nine were Amy group subjects and one was from the sham group. The final $n$ values for each group are indicated in the figure captions and tables. A $z$ test conducted on the two proportions revealed that significantly more mice were lost after surgical procedures in the Amy group than either Acb or sham groups in experiment 1 ( $z$ values $>3.45$; $p$ values $<0.05$ ), as well as more Amy-lesioned subjects than either Acb or Sham subjects in experiment 2 ( $z$ values $>3.9$; $p$ values $<0.05$ ).

\section{Histological verification of lesions}

Histological verification was performed on the remaining subjects. All histological analyses were performed blind to conditioning subgroup assignment and final test outcome. A total of 41 subjects were removed from the final data analyses (Table 1). Of those removed in experiment 1, eight subjects had lesions that either extended or were localized outside the inclusion sites. For experiment 2, data from 22 subjects with lesions that extended outside the inclusion boundaries were excluded. For experiment 3, 11 subjects had lesions that either encompassed both AcbC and AcbSh or had mixed AcbC and AcbSh lesions between hemispheres. Data from these subjects were excluded. Although of potential interest in relation to neural modulation of conditioned reinforcement and pavlovian approach behaviors (for review, see Everitt and Robbins, 2005), we were unable to separate contributions of the BLA and CE on the acquisition or expression of ethanol CPP because most subjects had lesions encompassing portions of both regions (experiment 1, 11 of 14 subjects; experiment 2, 25 of 32 subjects). Schematic diagrams are shown in Figure 1. 
A

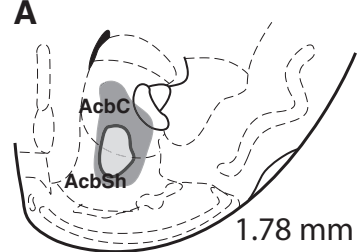

B
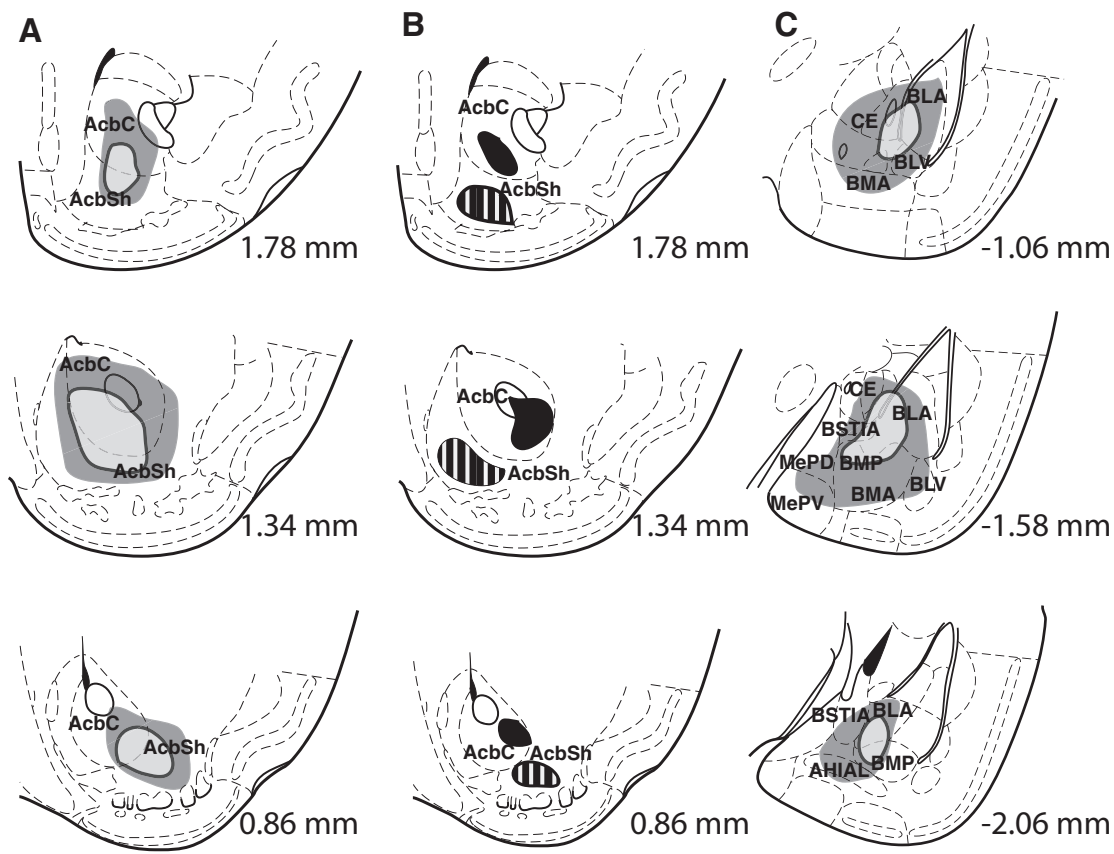

\section{$=$ Extent of maximum lesion}

$=$ Extent of maximum AcbC lesion

$\square=$ Extent of minimum lesion

\|I = Extent of maximum AcbSh lesion

Figure 1. Representative diagram of Acb and Amy lesions from bregma. $A$, The extent of maximum (dark gray) and minimum (light gray) Acb lesions in experiments 1 and 2. B, Maximum AcbSh (striped) and AcbC (black) lesions in experiment 3. C, The extent of maximum (dark gray) and minimum (light gray) Amy and Uni lesions in experiments 1 and 2.

\section{Pretest}

A pretest was conducted before conditioning for experiments 1 and 3 and for one replication of experiment 2 to examine initial biases for the conditioned stimuli (Fig. 2). Surgical procedures or lesions of the Acb or Amy in experiment 1 did not affect initial biases for the grid or hole floors as measured during a pretest (Fig. $2 A$ ). These findings were supported by a two-way ANOVA (conditioning subgroup by lesion site), which yielded no significant main effects or interaction. Furthermore, the site of the sham lesion did not affect initial bias as demonstrated by a lack of significant interaction or main effect of lesion site on pretest results. Lesion and sham subjects had similar levels of activity during the pretest (Table 2). Additionally, basal activity levels were not different between the sham groups.

Pretest results in experiments 2 (replicate 1) and 3 replicated previous findings (Cunningham et al., 2003) in that nonlesioned subjects did not display an unconditioned bias for one floor type, nor did lesion group assignment affect this lack of bias (Fig. $2 B, C)$. This conclusion was supported by two-way ANOVAs (conditioning subgroup by lesion site) that showed no significant main effects or interaction. Because of a procedural error, subjects in the second replicate of experiment 2 were exposed to a habituation session instead of a pretest. To determine whether development of CPP differed between the experiment 2 replicates that did (replicate 1) and did not (replicate 2) receive a pretest, a two-way ANOVA (replication by conditioning subgroup) was performed on preference test results in the sham group and in the Amy group. The sham replicates in experiment 2 did not differ, suggesting that the pretest performed in the first replication did not affect later expression of preference. Moreover, the Amy replicates in experiment 2 did not differ, suggesting that postconditioning lesions did not induce an effect of the pretest on later preference expression. Thus, the two replicates were combined for the preference test analyses described in the next section. Additionally, as expected, there were no activity differences between assigned lesion groups in experiment 2 or in experiment 3 (Table 2). Overall, because no differences in initial preference or activity were observed during the pretest, differences observed during the final test can be attributed to the effects of conditioning and preconditioning or postconditioning lesions on CPP.

\section{Preference testing}

Lesion effects on acquisition and expression of ethanol CPP

In experiment 1 , the roles of the Acb and Amy in the acquisition and expression of ethanol CPP were examined. After ethanol place conditioning, sham subjects expressed a strong place preference (Fig. $3 A$ ). However, Acb and Amy groups did not express a place preference, i.e., there was no difference between Grid + and Grid- conditioning subgroups after ethanol place conditioning. Moreover, both lesion groups differed significantly from the sham control group. Thus, lesions of the Acb or Amy prevented the acquisition and/or expression of ethanol-induced CPP.

A two-way (lesion group by conditioning subgroup) ANOVA performed on test session data revealed a main effect of conditioning subgroup (Grid+ vs Grid-) $\left(F_{(1,50)}\right.$ $=35.8 ; p<0.001)$ as well as a significant interaction $\left(F_{(2,50)}=\right.$ $9.0 ; p<0.001)$. The main effect of lesion was not significant. Post hoc analyses of the interaction revealed a significant difference between conditioning subgroups in the sham group (Bonferroni's corrected $p<0.001)$ that was not observed in either the Acb or the Amy group ( $p$ values $>0.05$ ). Furthermore, two-way (lesion group by conditioning subgroup) ANOVAs between pairs of specific lesion groups suggested that the Acb group differed from the sham group and that the Amy group differed from the sham group $(F$ values $>9.5$; $p$ values $<0.01$ ), although the Acb and the Amy groups did not differ from each other. A two-way ANOVA of sham site by conditioning subgroup showed no effect of sham lesion site on the magnitude of preference expressed in the sham group.

\section{Lesion effects on expression of ethanol CPP}

Contrary to findings in experiment 1 , which suggested a role for both Amy and Acb, the findings in experiment 2 suggested that expression of ethanol CPP was solely dependent on a fully intact Amy (Fig. 3B). After ethanol conditioning, surgery, and recovery, strong place preference was observed in the sham and Acb groups. However, unilateral Amy lesions (Uni group) partially reduced expression of ethanol place preference and bilateral Amy lesions completely disrupted expression of ethanol CPP.

These findings were supported by a two-way ANOVA (lesion group by conditioning subgroup) that revealed a main effect of lesion group $\left(F_{(3,127)}=5.0 ; p<0.01\right)$, conditioning subgroup $\left(F_{(1,127)}=68.4 ; p<0.001\right)$, and a significant interaction $\left(F_{(3,127)}\right.$ $=13.0 ; p<0.001)$. Post hoc analysis of the interaction showed a 
significant effect of conditioning subgroup in the sham, Acb, and Uni groups (Bonferroni's corrected $p$ values $<0.05$ ) but not in the Amy group $(p>0.05)$. Comparisons between pairs of lesion groups (lesion group by conditioning subgroup ANOVAs) found that groups sham and Acb did not differ $(p>0.1)$. However, the sham group did differ from the Uni group $\left(F_{(1,80)}=12.4, p<0.01\right)$ and from the Amy group $\left(F_{(1,85)}=45.3 ; p<0.001\right)$. Furthermore, the Amy group was significantly different from the Acb group $\left(F_{(1,47)}\right.$ $=11.4 ; p<0.01)$, and the Amy group showed a trend toward a difference compared with the Uni group $\left(F_{(1,55)}=3.4\right.$; $p=0.072)$. Groups Acb and Uni did not differ $(p>0.2)$.

A series of analyses were performed to examine extraneous factors that might have contributed to these findings. Site of the sham lesions had no effect as confirmed by a two-way ( sham site by conditioning subgroup) ANOVA on combined replicates. Additionally, because the effects of Amy and sham lesions on expression of ethanol CPP were performed in two replicates, separate two-way (replication by conditioning subgroup) ANOVAs were performed for each brain area. These analyses showed that the effects of Amy or sham lesions did not change across replicates ( $p$ values $>0.1$ ), suggesting similar effects in both replicates. Furthermore, a two-way (hemisphere by conditioning subgroup) ANOVA of the Uni group found no evidence that hemisphere lesioned (left vs right) contributed to effects on preference observed. Overall, these analyses indicate that preference differences between lesion groups were attributable to the brain area lesioned and not to procedural or surgical differences.

\section{Specific accumbens core versus shell lesion effects on expression of} ethanol CPP

Examination of the specific contributions of the Acb core and shell revealed differences in their impact on expression of ethanol CPP (Fig. 4). All lesion groups expressed similar levels of preference during the first half of the test session. However, lesions of the AcbC disrupted expression during the last $30 \mathrm{~min}$ of the test session, whereas mice with AcbSh lesions expressed a preference similar to that of the sham group.

A two-way ANOVA (conditioning subgroup by lesion group) conducted on the first $30 \mathrm{~min}$ of the test session revealed a main effect of conditioning subgroup $\left(F_{(1,31)}=65.5 ; p<0.001\right)$ but no main effect of lesion group or interaction. However, the same analysis conducted on the last $30 \mathrm{~min}$ of the test revealed a main effect of conditioning subgroup $\left(F_{(1,31)}=22.7 ; p<0.001\right)$ and a significant interaction $\left(F_{(2,31)}=3.3 ; p=0.05\right)$. There was no main effect of lesion during the last half of the test. Post hoc analyses of the interaction demonstrated a significant preference in both the sham and AcbSh groups (Bonferroni's corrected $p$ values $<0.001$ ) but not in the AcbC group. Comparisons between pairs of lesion groups found that the sham and AcbSh groups did not differ in level of preference expressed during the last $30 \mathrm{~min}$ $(p>0.7)$. However, the magnitude of preference expressed was significantly different between the sham and AcbC groups $\left(F_{(1,21)}\right.$ $=7.8 ; p<0.02)$, and there was a trend toward a significant

\section{Pretest}
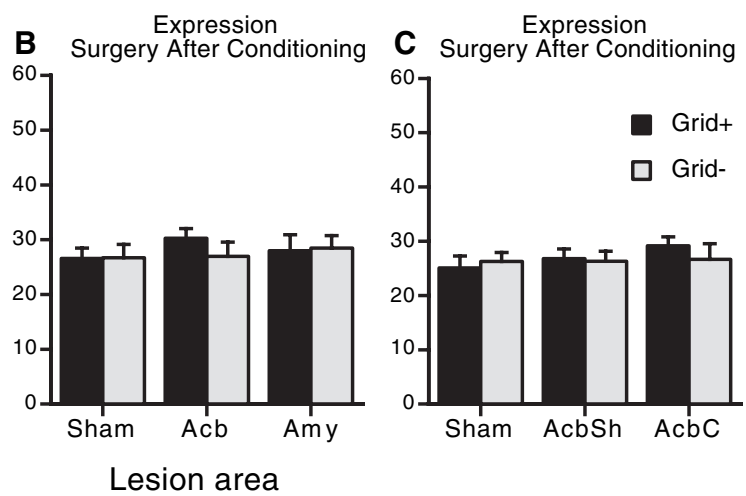
$0.067)$. The sham lesion area did not contribute to these differences, because there were no effects of sham site on preference expressed during the first or last $30 \mathrm{~min}$ of the test session.

\section{Conditioning activity}

For ease of presentation, conditioning activity data were averaged across trials to create single means for the CS + and CS - trials. As in previous studies in DBA/2J mice (Gremel and Cunningham, 2007; Cunningham et al., 2006b), ethanol given immediately before the conditioning trial induced increases in locomotor activity (for group means and statistical comparisons, see Table 2). In experiment 1, lesions of the Amy only slightly reduced this ethanol-induced activation. Ethanol also induced large increases in locomotor activity when administered before conditioning trials in experiments 2 and 3. However, in experiment 2, mean activity in the Uni group was slightly lower than other lesion groups, reflecting sampling differences among groups before lesion administration. There was no effect of replication in a separate analysis of the Amy and sham groups. In experiment 3, groups did not differ in their response to ethanol before the lesion was induced (Table 2).

\section{Test activity}

A history of ethanol treatment or conditioning procedures differentially affected test activity levels between lesion groups (for group means and statistical comparisons, see Table 2). Amy- and Acb-lesioned subjects had significantly higher test activity levels than the sham group in experiments 1 and 2. Moreover, unilateral Amy lesions before testing increased activity levels relative to sham lesions in experiment 2. In experiment 2, there was also a significant lesion by replication interaction in a separate analysis of the Amy and sham groups $\left(F_{(1,85)}=12.1 ; p=0.001\right)$. This interaction was attributable to slightly higher test activity levels in the Amy group during the second replication (increase of $\sim 10.6$ counts/min). In experiment 3, test activity levels did not differ between lesion groups during the first $30 \mathrm{~min}$ of the test session. However, during the last portion of the test, subjects with lesions 
Table 2. Locomotor activity

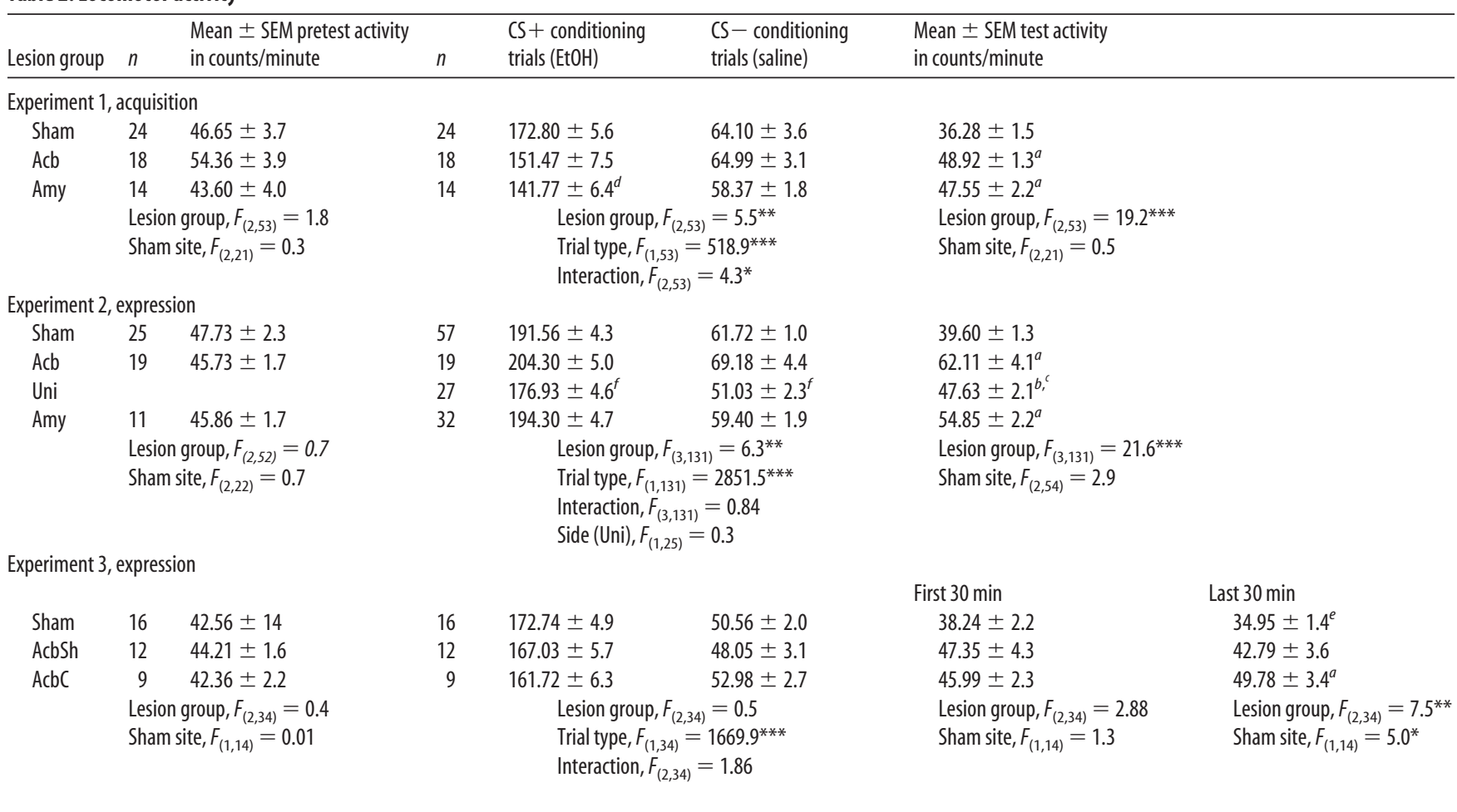

${ }^{*} p<0.05,{ }^{* *} p<0.01,{ }^{* * *} p<0.001,{ }^{* * * *} p<0.0001$ ( $p$ values for all group comparisons are Bonferroni's corrected).

${ }^{a}$ Difference from sham, $p<0.001$.

${ }^{b}$ Difference from $A c b, p=0.01$.

'Difference from sham, $p<0.05$.

${ }^{d}$ Difference from sham, $p<0.01$.

${ }^{e}$ Sham site AcbC and sham site AcbSh group means significantly different, $p<0.05$.

${ }^{f}$ Group Uni mean activity different from sham, Acb, and Amy, $p<0.05$.

of the AcbC had significantly higher levels of activity than sham subjects.

\section{Discussion}

These experiments are the first to reveal the functional importance of the Acb and Amy in the learning and expression of behavior controlled by a cue previously paired with ethanol. Lesions of Acb and Amy disrupted acquisition, whereas lesions of the Amy disrupted expression of ethanol CPP. CPP allows for investigation of ethanol-conditioned behaviors, without the confounding effects of ethanol on reinforced responding observed in self-administration models. Furthermore, CPP has the unique advantage over self-administration procedures in the measurement of conditioned behavior, because the response controlled by the conditioned cue has never produced the primary reinforcer (i.e., ethanol). The response measured during expression testing is approach and maintenance of contact with the previously drug-paired cue, without administration of the conditioning drug. Although theoretically pavlovian approach behavior, conditioned reinforcement, and conditioned incentive may all be operating in CPP, it is likely that only the latter two processes are involved in our procedure because animals are responding to a tactile cue in the dark (Cunningham et al., 2006b).

Lesion-induced deficits in the acquisition of CPP could be attributed to a disruption in the primary rewarding effects of ethanol or to a decrement in learning the association between the cue and ethanol (Bardo and Bevins, 2000; Cunningham et al., 2006a). However, lesions made before conditioning also affect CPP expression, hence not allowing for separation between the neural areas involved in the learning versus the manifestation of the behavior. For this reason, in experiments 2 and 3, lesions were administered after conditioning. Lesion effects on expression of CPP may reflect a decrease in the conditioned value of the cue, an inability to retrieve the memory of the association between the drug and cue, or a decrement in the learning or performance of the approach response. Although the present studies do not distinguish among these possibilities, they strongly suggest that different neural areas mediate the acquisition and expression of behavior controlled by ethanol-paired stimuli.

\section{Acquisition of ethanol CPP is dependent on Acb and Amy}

In experiment 1 , lesions of the Acb and Amy before conditioning interfered with the expression of ethanol CPP (Fig. 3). Unlike other drugs of abuse such as cocaine and amphetamine, there has been little direct evidence for involvement of the Acb or the Amy in the acquisition of the conditioned effects of ethanol. Previous work found that intra-Acb dopamine depletion by 6-hydroxydopmaine (6-OHDA) lesions did not alter the acquisition of ethanol self-administration (Myers and Quarfordt, 1991; Lyness and Smith, 1992; Rassnick et al., 1993), suggesting that the Acb may not be necessary for the primary reinforcing effects of ethanol. However, the current findings are more in line with previous studies examining the effect of Acb and Amy lesions on the acquisition of CPP induced by other abused drugs. For example, excitotoxic lesions of the Amy prevented the acquisition of cocaine CPP (Brown and Fibiger, 1993), and excitotoxic lesions of the lateral Amy attenuated amphetamine CPP (Hiroi and White, 1991). Furthermore, lesions of the Acb disrupted 
amphetamine CPP (Olmstead and Franklin, 1996) but had no effect on cocaine CPP (Spyraki et al., 1982). There have been mixed results as to the role of the Acb and Amy in morphine CPP. For example, there have been reports of 6-OHDA and electrolytic lesions of the Acb disrupting acquisition of morphine CPP (Kelsey et al., 1989; Shippenberg et al., 1993; White et al., 2005), whereas Olmstead and Franklin (1997) found that excitotoxic lesions of the Acb or Amy failed to disrupt acquisition of morphine CPP.

\section{Ethanol CPP is expressed through an Amy-dependent mechanism}

In contrast to findings from the acquisition study (experiment 1), only lesions of the Amy had an effect on expression of ethanol CPP (experiment 2). Bilateral lesions performed after conditioning completely disrupted $\mathrm{CPP}$, whereas unilateral lesions attenuated expression of ethanol conditioned behavior (Fig. 3). Of particular interest, the findings of Amy involvement in ethanol CPP are in parallel to studies examining ethanol reinforcement in self-administration procedures (Roberts et al., 1996; Heyser et al., 1999; Schroeder et al., 2003).

In contrast to the Amy findings, lesions of the Acb did not affect initial expression of CPP. This finding is not only at odds with studies demonstrating the importance of the Acb in the primary reinforcing effects of ethanol (for review, see McBride and $\mathrm{Li}, 1998$ ) but is also divergent from findings examining contributions of the $\mathrm{Acb}$ in ethanol-seeking using a selfadministration procedure that suggested a role for the Acb (particularly the core region) in stimulus processing of alcohol-related cues (Samson et al., 1999; Czachowski et al., 2001; Samson and Chappell, 2003, 2004; Czachowski, 2005). It may be that control over ethanol-seeking behavior by a conditioned cue only involves some of the same areas, mainly the Amy, as those recruited in an ethanol self-administration model.

However, one issue in comparing our studies is the difference in time elapsed between conditioning and testing in experiment 1 ( $1 \mathrm{~d}$ ) versus experiments 2 and 3 (13 d). It is possible, for example, that timedependent compensatory mechanisms may have masked an effect of Acb lesions on expression of ethanol CPP in experiments 2 and 3. Alternatively, Acb-lesioned, compared with Amy-lesioned, mice may be more sensitive to postconditioning changes in the strength of $\mathrm{CPP}$ over time. However, a cross-experiment comparison of the sham groups (experiment by conditioning subgroup ANOVA) showed no significant differences across experiments, suggesting that the memory of the pavlovian association controlling ethanol CPP is not affected by a delay of $13 \mathrm{~d}$ before testing. Although we cannot completely dismiss a possible role of postconditioning delay, Bonferroni's corrected. Bonferroni's corrected.

\section{Preference test}

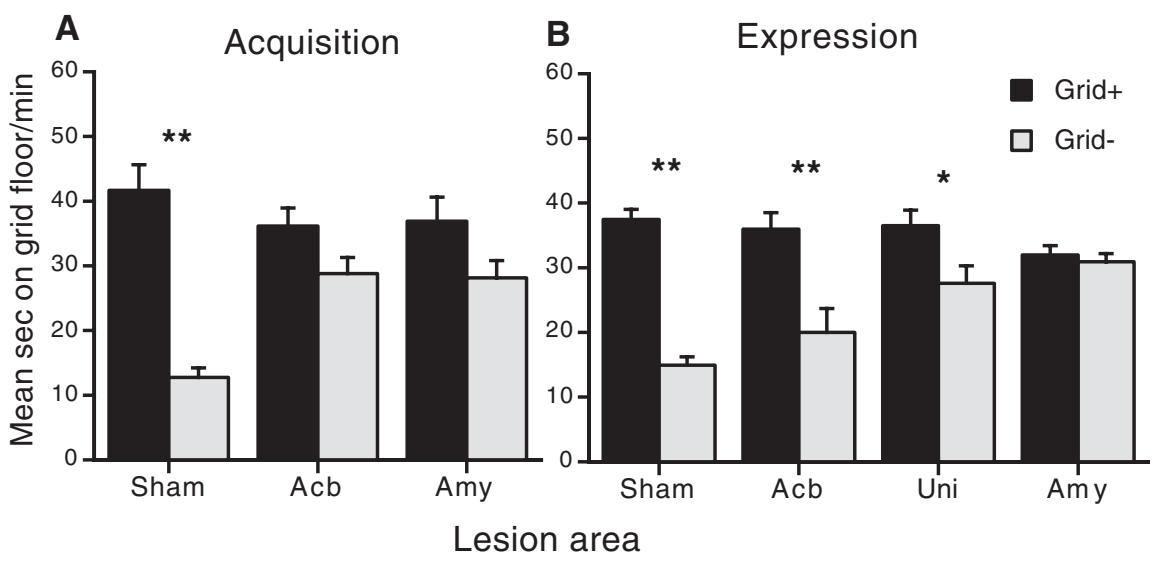

Figure 3. Lesions of the Acb and Amy disrupt acquisition, but only lesions of the Amy block expression of ethanol CPP. Mean \pm SEM seconds per minute spent on the grid floor during the $30 \mathrm{~min}$ test session. $\boldsymbol{A}$, In the acquisition experiment, subjects received sham lesions or lesions of the Acb or Amy before ethanol place conditioning. Grid + and Grid - conditioning subgroup $n$ values are as follows: sham, $n=12$ and 12; $A c b, n=9$ and 9; and Amy, $n=6$ and 8. B. However, subjects in the expression experiment received sham lesions or lesions of the $A c b$, unilateral $A m y$, or Amy after conditioning but before preference testing. Grid + and Grid - conditioning subgroup $n$ values are as follows: sham, $n=29$ and 28; $A c b, n=10$ and 9; Uni, $n=13$ and 14; and Amy, $n=$ 17 and 15. Difference between conditioning subgroups Grid + and Grid-: ${ }^{*} p<0.05$, Bonferroni's corrected; ${ }^{* *} p<0.001$,

\section{Preference test}

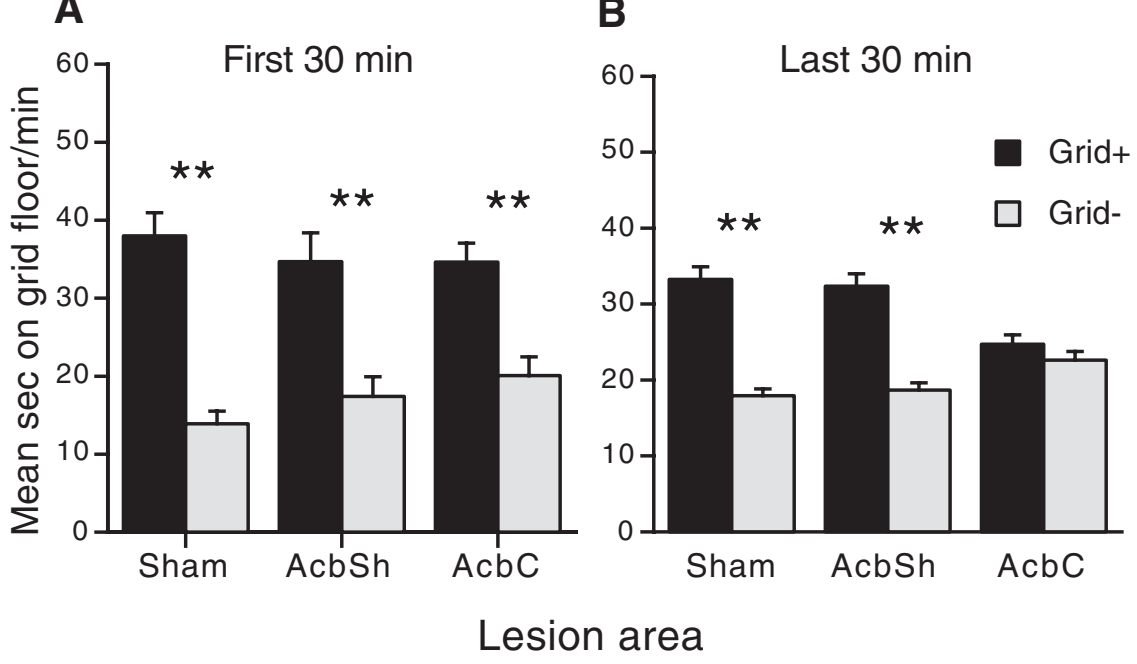

Figure 4. Lesions of the $A c b C$ disrupt expression of ethanol CPP during the last half of the test session. Mean \pm SEM seconds per minute spent on the grid floor during the first and last 30 min of the test session. $A$, First 30 min of the test session. The sham, $A c b S h$, and $A c b C$ groups that were lesioned after ethanol place conditioning all express strong ethanol CPP. $B$, However, during that last 30 min of the test session, subjects in the $A c b C$ group no longer expressed ethanol CPP, whereas preference in the AcbSh and sham groups was still significant. Grid + and Grid - conditioning subgroups $n$ values are, respectively, as follows: sham, $n=$ 8 and $8 ; A c b S h, n=6$ and 6 ; and $A c b C, n=4$ and 5. Difference between conditioning subgroups Grid + and Grid $-:{ }^{* *} p<0.01$,

the similarity among sham groups argues against this difference as an important determinant of the difference in the outcomes of the acquisition and expression experiments.

\section{Lesions of the AcbC facilitate loss of responding}

In experiments 1 and 2, mice in the Acb group had lesions that extended into both the AcbSh and AcbC, not allowing for possible behavioral separation based on lesion location. Thus, experiment 3 was performed to ask whether subjects with lesions focused in the AcbC would differ from AcbSh- and sham-lesioned 
subjects. We found that AcbSh and sham groups displayed a similar magnitude preference throughout the 60-min expression test. Although lesions of the AcbC had no effect on initial expression of ethanol CPP, these mice showed a more rapid loss of responding (Fig. $4 \mathrm{~B}$ ), possibly implicating the AcbC in the inhibitory learning believed to occur when the context is no longer paired with drug during CPP extinction.

\section{Overlap with areas activated by ethanol-paired cue exposure}

As expected, there is some overlap between the neural areas mediating the acquisition and expression of ethanol CPP and those activated by exposure to a cue paired previously with ethanol. For example, in a ethanol reinstatement model using a discriminative stimulus task, increased Fos expression was observed in the Acb (Dayas et al., 2007), in addition to the Acb, BLA, and CE in a separate study (Zhao et al., 2006). Furthermore, recent findings from an ethanol cue-induced reinstatement study found increases in BLA and CE activation in response to a discrete cue (Radwanska et al., 2007). In a study that used a pavlovian conditioning procedure similar to ours, the CS+ elicited increased Fos expression in the extended Amy and anterior VTA, but no conditioned increase was observed in the Acb (Hill et al., 2007). Although the procedural differences responsible for the above discrepancies are unknown, these findings generally suggest that the Amy and Acb play major roles in the processing of ethanolpredictive environmental stimuli and mediation of ethanolseeking behavior as indexed by CPP and ethanol reinstatement models.

\section{The role of the Acb and Amy in locomotor activity}

Present findings also shed light on the neural areas governing ethanol-induced activation and test activity in mice that are hypothesized to overlap with those involved in its reinforcing effects (Phillips and Shen, 1996; Boehm et al., 2002). In experiment 1, only lesions of the Amy attenuated ethanol-induced stimulation during conditioning trials. Although the differences in activity were relatively minor, subjects with Amy and Acb lesions showed higher levels of activity during the drug-free test. Although level of activation can affect preference (Gremel and Cunningham, 2007), we do not believe that the differences in activity observed in the present studies were sufficient to significantly alter the observed CPP because Acb-lesioned subjects expressed a preference similar to sham subjects.

In summary, our data suggest that the Amy and Acb influence the acquisition of ethanol CPP, whereas the Amy modulates CPP expression. Overall, these findings suggest that the neural mechanisms underlying ethanol CPP mirror some mechanisms recruited during ethanol self-administration but involve seeking mechanisms also engaged by other drugs of abuse. Although the use of electrolytic lesions in the present study prevents the interpretation of direct effects on nuclei, the results strongly implicate these areas in the mediation of ethanol CPP. Future work will aim to delineate the specific neurotransmitters in each area contributing to the acquisition, expression, and extinction of this behavior.

\section{References}

Bardo MT, Bevins RA (2000) Conditioned place preference: what does it add to our preclinical understanding of drug reward? Psychopharmacology (Berl) 153:31-43.

Bechtholt AJ, Cunningham CL (2005) Ethanol-induced conditioned place preference is expressed through a ventral tegmental area dependent mechanism. Behav Neurosci 119:213-223.

Boehm SL, Piercy MM, Bergstrom HC, Phillips TJ (2002) Ventral tegmental area region governs $\mathrm{GABA}(\mathrm{B})$ receptor modulation of ethanol-stimulated activity in mice. Neuroscience 115:185-200.

Brodie MS, Shefner SA, Dunwiddie TV (1990) Ethanol increases the firing rate of dopamine neurons of the rat ventral tegmental area in vitro. Brain Res 508:65-69.

Brodie MS, Pesold C, Appel SB (1999) Ethanol directly excites dopaminergic ventral tegmental area reward neurons. Alcohol Clin Exp Res 23:1848-1852.

Brown EE, Fibiger HC (1993) Differential effects of excitotoxic lesions of the amygdala on cocaine-induced conditioned locomotion and conditioned place preference. Psychopharmacology (Berl) 113:123-130.

Ciccocioppo R, Angeletti S, Weiss F (2001) Long-lasting resistance to extinction of response reinstatement induced by ethanol-related stimuli: role of genetic ethanol preference. Alcohol Clin Exp Res 25:1414-1419.

Ciccocioppo R, Martin-Fardon R, Weiss F (2002) Effect of selective blockade of $\mu 1$ or $\delta$ opioid receptors on reinstatement of alcohol-seeking behavior by drug-associated stimuli in rats. J Neurosci 27:391-399.

Ciccocioppo R, Lin D, Martin-Fardon R, Weiss F (2003) Reinstatement of ethanol-seeking behavior by drug cues following single versus multiple ethanol intoxication in the rat: effects of naltrexone. Psychopharmacology (Berl) 168:208-215.

Corbit LH, Janak PH (2007) Ethanol-associated cues produce general pavlovian-instrumental transfer. Alcohol Clin Exp Res 31:766-774.

Cunningham CL (1994) Modulation of ethanol reinforcement by conditioned hyperthermia. Psychopharmacology (Berl) 115:79-85.

Cunningham CL (1998) Drug conditioning and drug-seeking behavior. In: Learning and behavior therapy (O'Donohue W, ed), pp 518-544. Boston: Allyn and Bacon.

Cunningham CL, Niehus JS, Noble D (1993) Species difference in sensitivity to ethanol's hedonice effects. Alcohol 10:97-102.

Cunningham CL, Okorn DM, Howard C (1997) Interstimulus interval determines whether ethanol produces conditioned place preference or aversion in mice. Animal Learn Behav 25:31-42.

Cunningham CL, Ferree NK, Howard MA (2003) Apparatus bias and place conditioning with ethanol in mice. Psychopharmacology (Berl) 170:409-422.

Cunningham CL, Gremel CM, Groblewski PA (2006a) Drug-induced conditioned place preference and aversion in mice. Nat Protoc 1:1662-1670.

Cunningham CL, Patel P, Milner L (2006b) Spatial location is critical for conditioning place preference with visual but not tactile stimuli. Behav Neurosci 120:1115-1132.

Czachowski CL (2005) Manipulations of serotonin function in the nucleus accumbens core produce differential effects on ethanol and sucrose seeking and intake. Alcohol Clin Exp Res 29:1146-1155.

Czachowski CL, Chappell AM, Samson HH (2001) Effects of raclopride in the nucleus accumbens on ethanol seeking and consumption. Alcohol Clin Exp Res 25:1431-1440.

Dayas CV, Liu X, Simms JA, Weiss F (2007) Distinct patterns of neural activation associated with ethanol seeking: effects of naltrexone. Biol Psychiatry 61:979-989.

Di Chiara G, Imperato A (1988) Drugs abused by humans preferentially increase synaptic dopamine concentrations in the mesolimbic system of freely moving rats. Proc Natl Acad Sci USA 85:5274-5278.

Di Ciano P, Everitt BJ (2005) Neuropsychopharmacology of drug seeking: Insights from studies with second-order schedules of drug reinforcement. Eur J Pharmacol 526:186-198.

Everitt BJ, Robbins TW (2005) Neural systems of reinforcement for drug addiction: from actions to habits to compulsion. Nat Neurosci 8:1481-1489.

Fidler TL, Bakner L, Cunningham CL (2004) Conditioned place aversion induced by intragastric administration of ethanol in rats. Pharmacol Biochem Behav 77:731-743.

Gatto GJ, McBride WJ, Murphy JM, Lumeng L, Li TK (1994) Ethanol selfinfusion into the ventral tegmental area by alcohol-preferring rats. Alcohol 11:557-564.

Gessa GL, Muntoni F, Collu M, Vargiu L, Mereu G (1985) Low doses of ethanol activate dopaminergic neurons in the ventral tegmental area. Brain Res 348:201-203.

Gremel CM, Cunningham CL (2007) Role of test activity in ethanolinduced disruption of place preference expression in mice. Psychopharmacology (Berl) 191:195-202.

Heyser CJ, Roberts AJ, Schulteis G, Koob GF (1999) Central administration 
of an opiate antagonist decreases oral ethanol self-administration in rats. Alcohol Clin Exp Res 23:1468-1476.

Hill KG, Ryabinin AE, Cunningham CL (2007) FOS expression induced by an ethanol-paired conditioned stimulus. Pharmacol Biochem Behav $87: 208-221$.

Hiroi N, White NM (1991) The lateral nucleus of the amygdala mediates expression of the amphetamine-produced conditioned place preference. J Neurosci 11:2107-2116.

Hyytiä P, Koob GF (1995) GABAA receptor antagonism in the extended amygdala decreases ethanol self-administration in rats. Eur J Pharmacol 283:151-159.

Katner SN, Weiss F (1999) Ethanol-associated olfactory stimuli reinstate ethanol-seeking behavior after extinction and modify extracellular dopamine levels in the nucleus accumbens. Alcohol Clin Exp Res 23:1751-1760.

Kelsey JE, Carlezon WA, Falls WA (1989) Lesions of the nucleus accumbens in rats reduce opiate reward but do not alter context-specific opiate tolerance. Behav Neurosci 103:1327-1334.

Koob GF, Le Moal M (2006) Neurobiology of addiction. London, UK: Academic.

Krank MD (1989) Environmental signals for ethanol exposure enhance free-choice ethanol consumption. Behav Neurosci 103:365-372.

Krank MD (2003) Pavlovian conditioning with ethanol: sign-tracking (autoshaping), conditioned incentive, and ethanol self-administration. Alcohol Clin Exp Res 27:1592-1598.

Krank MD, Wall AM (1990) Cue exposure during a period of abstinence reduces the resumption of operant behavior for oral ethanol reinforcement. Behav Neurosci 104:725-733.

Lyness WH, Smith FL (1992) Influence of dopaminergic and serotonergic neurons on intravenous ethanol self-administration in the rat. Pharmacol Biochem Behav 42:187-192.

McBride WJ, Li TK (1998) Animal models of alcoholism: neurobiology of high alcohol-drinking behavior in rodents. Crit Rev Neurobiol 12:339-369.

Myers RD, Quarfordt SD (1991) Alcohol drinking attenuated by sertraline in rats with 6-OHDA or 5,7-DHT lesions of the N. accumbens: a caloric response? Pharmacol Biochem Behav 40:923-928.

Nie H, Janak PH (2003) Comparison of reinstatement of ethanol- and sucrose-seeking by conditioned stimuli and priming injections of allopregnanolone after extinction in rats. Psychopharmacology (Berl) 168:222-228.

Olmstead MC, Franklin KB (1996) Differential effects of ventral striatal lesions on the conditioned place preference induced by morphine or amphetamine. Neuroscience 71:701-708.

Olmstead MC, Franklin KB (1997) The development of a conditioned place preference to morphine: effects of microinjections into various CNS sites. Behav Neurosci 111:1324-1334.

Paxinos G, Franklin KBJ (2001) The mouse brain in stereotaxic coordinates. San Diego: Academic.

Phillips TJ, Shen EH (1996) Neurochemical bases of locomotion and ethanol stimulant effects. Int Rev Neurobiol 39:243-282.

Radwanska K, Wrobel E, Korkosz A, Rogowski A, Kostowski W, Bienkowski P, Kaczmarek L (2007) Alcohol relapse induced by discrete cues activates components of AP-1 transcription factor and ERK pathway in the rat basolateral and central amygdala. Neuropsychopharmacology, in press.

Rassnick S, Stinu L, Koob GF (1993) The effects of 6-hydroxydopamine lesions of the nucleus accumbens and the mesolimbic dopamine system on oral self-administration of ethanol in the rat. Brain Res 623:16-24.

Roberts AJ, Cole M, Koob GF (1996) Intra-amygdala muscimol decreases operant ethanol self-administration in dependent rats. Alcohol Clin Exp Res 20:1289-1298.

Rodd ZA, Melendez RI, Bell RL, Kuc KA, Zhang Y, Murphy JM, McBride WJ (2004) Intracranial self-administration of ethanol within the ventral tegmental area of male Wistar rats: evidence for involvement of dopamine neurons. J Neurosci 24:1050-1057.

Samson HH, Hodge CW (1996) Neurobehavioral regulation of ethanol intake. In: Pharmacological effects of ethanol on the nervous system (Deitrich R, Erwin V, eds). Boca Raton, FL: CRC.

Samson HH, Chappell AM (2003) Dopaminergic involvement in medial prefrontal cortex and core of the nucleus accumbens in the regulation of ethanol self-administration: a dual-site microinjection study in the rat. Physiol Behav 79:581-590.

Samson HH, Chappell AM (2004) Effects of raclopride in the core of the nucleus accumbens on ethanol seeking and consumption: the use of extinction trials to measure seeking. Alcohol Clin Exp Res 28:544-549.

Samson HH, Chappell AM, Slawecki C, Hodge C (1999) The effects of microinjection of $d$-amphetamine into the $\mathrm{n}$. accumbens during the late maintenance phase of an ethanol consumption bout. Pharmacol Biochem Behav 63:159-165.

Schauwecker PE (2005) Susceptibility to excitotoxic and metabolic striatal neurodegeneration in the mouse is genotype dependent. Brain Res 1040:112-120.

Schauwecker PE, Steward O (1997) Genetic determinants of susceptibility to excitotoxic cell death: implications for gene targeting approaches. Proc Natl Acad Sci USA 94:4103-4108.

Schroeder JP, Olive F, Koenig H, Hodge CW (2003) Intra-amygdala infusion of the NPY Y1 receptor antagonist BIBP 3226 attenuates operant ethanol self-administration. Alcohol Clin Exp Res 27:1884-1891.

Shippenberg TS, Bals-Kubik R, Herz A (1993) Examination of the neurochemical substrates mediating the motivational effects of opioids: role of the mesolimbic dopamine system and D-1 vs. D-2 dopamine receptors. J Pharmacol Exp Ther 265:53-59.

Spyraki C, Fibiger HC, Phillips AG (1982) Cocaine-induced place preference conditioning: lack of effects of neuroleptics and 6-hydroxydopamine lesions. Brain Res 253:195-203.

Tzschentke TM (2007) Measuring reward with the conditioned place preference (CPP) paradigm: update of the last decade. Addict Biol $12: 227-462$.

Weiss F (2005) Neurobiology of craving, conditioned reward and relapse. Curr Opin Pharmacol 5:9-19.

White NM, Chai SC, Hamdani S (2005) Learning the morphine conditioned cue preference: cue configuration determines effects of lesions. Pharmacol Biochem Behav 81:786-796.

Yoshimoto K, Ueda S, Kato B, Takeuchi Y, Kawai Y, Noritake K, Yasuhara M (2000) Alcohol enhances characteristic releases of dopamine and serotonin in the central nucleus of the amygdala. Neurochem Int 37:369-376.

Zhao Y, Dayas CV, Aujla H, Baptista MA, Martin-Fardon R, Weiss F (2006) Activation of group II metabotropic glutamate receptors attenuates both stress and cue-induced ethanol-seeking and modulates c-fos expression in the hippocampus and amygdala. J Neurosci 26:9967-9974.

Zironi I, Burattini C, Aicardi G, Janak PH (2006) Context is a trigger for relapse to alcohol. Behav Brain Res 167:150-155. 